Acta Crystallographica Section E

Structure Reports

Online

ISSN 1600-5368

\section{3,3'-Dibromo-1,1'-[ethylenedioxy- bis(nitrilomethylidyne)]dibenzene}

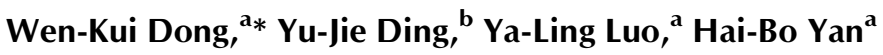 and Li Wang ${ }^{\mathrm{a}}$}

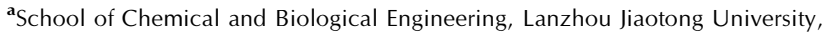
Lanzhou 730070, People's Republic of China, and 'bepartment of Biochemical Engineering, Anhui University of Technology and Science, Wuhu 241000, People's Republic of China

Correspondence e-mail: dongwk@mail.Izjtu.cn

Received 2 June 2008; accepted 4 July 2008

Key indicators: single-crystal X-ray study; $T=298 \mathrm{~K}$; mean $\sigma(\mathrm{C}-\mathrm{C})=0.005 \AA$; $R$ factor $=0.037 ; w R$ factor $=0.098 ;$ data-to-parameter ratio $=14.0$.

In the centrosymmetric title compound, $\mathrm{C}_{16} \mathrm{H}_{14} \mathrm{Br}_{2} \mathrm{~N}_{2} \mathrm{O}_{2}$, the intramolecular interplanar distance between the parallel benzene rings is 1.305 (3) $\AA$, while the intermolecular interplanar distance (between neighbouring molecules) is 3.463 (3) $\AA$, exhibiting obvious strong intermolecular $\pi-\pi$ stacking interactions.

\section{Related literature}

For related literature, see: Akine et al. (2006); Atwood \& Harvey (2001); Dong \& Duan (2008); Dong et al. (2007); Dong, Duan et al. (2008); Dong, Shi et al. (2008); Katsuki (1995); Sun et al. (2004).<smiles>Brc1cccc(/C=N/OCCO/N=C/c2cccc(Br)c2)c1</smiles>

\section{Experimental}

Crystal data

$\mathrm{C}_{16} \mathrm{H}_{14} \mathrm{Br}_{2} \mathrm{~N}_{2} \mathrm{O}_{2}$

$M_{r}=426.11$

Monoclinic, $P 2_{1} / n$ $\beta=93.523(2)^{\circ}$

$V=794.1(3) \AA^{3}$

$Z=2$

Mo $K \alpha$ radiation

$\mu=5.11 \mathrm{~mm}^{-1}$

$T=298(2) \mathrm{K}$

$0.42 \times 0.27 \times 0.15 \mathrm{~mm}$

Data collection

Siemens SMART 1000 CCD areadetector diffractometer

Absorption correction: multi-scan (SADABS; Sheldrick, 1996)

$T_{\min }=0.223, T_{\max }=0.514$

(expected range $=0.201-0.464)$

Refinement

$R\left[F^{2}>2 \sigma\left(F^{2}\right)\right]=0.037$

$w R\left(F^{2}\right)=0.097$

$S=1.04$

1400 reflections

3840 measured reflections 1400 independent reflections 1168 reflections with $I>2 \sigma(I)$ $R_{\text {int }}=0.043$

100 parameters

$\mathrm{H}$-atom parameters constrained

$\Delta \rho_{\max }=0.28 \mathrm{e} \AA^{-3}$

$\Delta \rho_{\min }=-0.49{\mathrm{e} \AA^{-3}}^{-3}$

Data collection: SMART (Siemens, 1996); cell refinement: SAINT (Siemens, 1996); data reduction: SAINT; program(s) used to solve structure: SHELXS97 (Sheldrick, 2008); program(s) used to refine structure: SHELXL97 (Sheldrick, 2008); molecular graphics: SHELXTL (Sheldrick, 2008); software used to prepare material for publication: SHELXTL.

This work was supported by the Foundation of the Education Department of Gansu Province (No. 0604-01) and the 'Qing Lan' Talent Engineering Funds of Lanzhou Jiaotong University (No. QL-03-01 A), which are gratefully acknowledged.

Supplementary data and figures for this paper are available from the IUCr electronic archives (Reference: WW2122).

\title{
References
}

Akine, S., Dong, W. K. \& Nabeshima, T. (2006). Inorg. Chem. 45, 4677-4684. Atwood, D. A. \& Harvey, M. J. (2001). Chem. Rev. 101, 37-52.

Dong, W. K., Chen, X., Wang, S. J., He, X. N., Wu, H. L. \& Yu, T. Z. (2007). Synth. React. Inorg. Met.-Org. Nano-Met. Chem. 37, 229-233.

Dong, W. K. \& Duan, J. G. (2008). J. Coord. Chem. 61, 781-788

Dong, W. K., Duan, J. G., Chai, L. Q., Liu, G. L. \& Wu, H. L. (2008). J. Coord. Chem. 61, 1306-1315.

Dong, W. K., Shi, J. Y., Zhong, J. K., Tian, Y. Q. \& Duan, J. G. (2008). Chin. J. Inorg. Chem. 28, 10-14.

Katsuki, T. (1995). Coord. Chem. Rev. 140, 189-214.

Sheldrick, G. M. (1996). SADABS. University of Göttingen, Germany.

Sheldrick, G. M. (2008). Acta Cryst. A64, 112-122.

Siemens (1996). SMART and SAINT. Siemens Analytical X-ray Systems Inc., Madison, Wisconsin, USA.

Sun, S. S., Stern, C. L., Nguyen, S. T. \& Hupp, J. T. (2004). J. Am. Chem. Soc. 126, 6314-6326. 


\section{supporting information}

Acta Cryst. (2008). E64, o1636 [doi:10.1107/S1600536808020692]

\section{3,3'-Dibromo-1,1'-[ethylenedioxybis(nitrilomethylidyne)]dibenzene \\ Wen-Kui Dong, Yu-Jie Ding, Ya-Ling Luo, Hai-Bo Yan and Li Wang}

\section{S1. Comment}

Schiff base compounds have played an important role in the development of coordination chemistry as they can readily form stable complexes with most metal ions (Dong \& Duan, 2008; Dong, Duan et al., 2008; Dong, Shi et al., 2008; Atwood \& Harvey, 2001). These complexes are very interesting in many fields, such as catalysis and enzymatic reactions (Akine et al., 2006), magnetism and molecular architectures (Sun et al., 2004; Katsuki, 1995). However, to the best of our knowledge, the complexes derived from the Schiff base 3,3'-dibromo-1,1'-[ethylenedioxybis(nitriloethylidyne)]dibenzene have never been reported so far. Information on the structure of the Schiff base compound will help us understand the interactions in the molecule so as to further design and synthesize complexes derived from this ligand. As a further investigation on such compounds, we report herein the synthesis and crystal structure of the Schiff base bisoxime compound 3,3'-dibromo-1,1'-[ethylenedioxybis(nitriloethylidyne)]dibenzene, shown in Fig. 1.

The X-ray crystallography reveals the title compound crystallizes in the monoclinic system, space group P2(1)/n with a $=4.5072$ (7) $\AA, \mathrm{b}=7.615$ (2) $\AA, \mathrm{c}=23.180$ (3) $\AA, \beta=93.523$ (2) ${ }^{\circ}$ and $Z=2$. The structure of the title compound consists of discrete $\mathrm{C}_{16} \mathrm{H}_{14} \mathrm{Br}_{2} \mathrm{~N}_{2} \mathrm{O}_{2}$ molecules in which all bond lengths are in normal ranges.

The molecule is disposed about a crystallographic centre of symmetry at the mid-point of the $\left(\mathrm{CH}_{2}-\mathrm{CH}_{2}\right)$ linkage adopting an anti-symmetrized conformation in which two benzaldoxime moieties adopt an extended form. Both intra- and inter-molecular hydrogen bonds are not observed in the title compound. The intramolecular plane-to-plane distance of the benzene rings was found to be $1.305 \AA$, while that of the intermolecular plane-to-plane distance (between neighbouring molecules) was found to be 3.463 (3) $\AA$, exhibiting obvious strong intermolecular $\pi$ - $\pi$ stacking interactions.

\section{S2. Experimental}

3,3'-Dibromo-1,1'-[ethylenedioxybis(nitriloethylidyne)]dibenzene was synthesized according to our previous work (Dong et al., 2007). To an ethanol solution (3 ml) of 3-bromo-benzaldehyde (398.7 mg, $2.15 \mathrm{mmol})$ was added dropwise an ethanol solution ( $2 \mathrm{ml}$ ) of 1,2-bis(aminooxy)ethane $(96.2 \mathrm{mg}, 1.04 \mathrm{mmol})$. The mixture solution was stirred at $328 \mathrm{~K}$ for $4 \mathrm{~h}$. After cooling to room temperature, the precipitate was filtered off, and washed successively with ethanol and ethanol-hexane mixture (1:4), respectively. The product was dried in vacuo to yield $366.0 \mathrm{mg}$ (Yield, 82.3\%) of colorless microcrystals; m.p. 363.5 - 365.5 K. Anal. Calcd. for $\mathrm{C}_{16} \mathrm{H}_{14} \mathrm{Br}_{2} \mathrm{~N}_{2} \mathrm{O}_{2}$ : C, 45.10; H, 3.31; N, 6.57. Found: C, 45.01; H, $3.20 ; \mathrm{N}, 6.43 \%$.

Single crystals were obtained by slow evaporation from a ethanol-acetone mixed solution of the title compound at room temperature.

\section{S3. Refinement}

Non- $\mathrm{H}$ atoms were refined anisotropically. $\mathrm{H}$ atoms were treated as riding atoms with distances $\mathrm{C}-\mathrm{H}=0.97\left(\mathrm{CH}_{2}\right), 0.93$ $\AA(\mathrm{CH})$, and $U_{\text {iso }}(\mathrm{H})=1.2 U_{\mathrm{eq}}(\mathrm{C})$. 


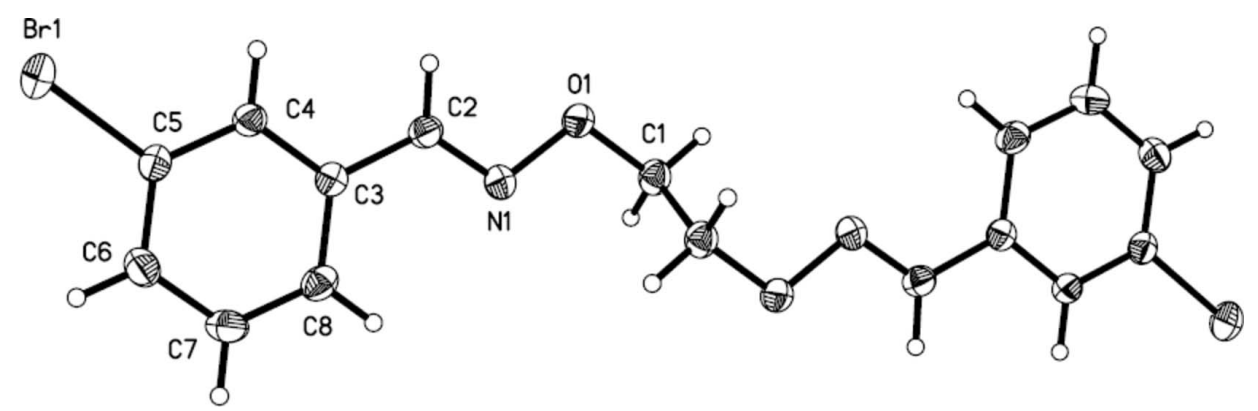

\section{Figure 1}

The molecular structure of the title compound with atom numbering scheme [Symmetry codes: $-x-1,-y,-z]$. Displacement ellipsoids for non-hydrogen atoms are drawn at the $30 \%$ probability level.

\section{3,3'-Dibromo-1,1'-[ethylenedioxybis(nitrilomethylidyne)]dibenzene}

\section{Crystal data}

$\mathrm{C}_{16} \mathrm{H}_{14} \mathrm{Br}_{2} \mathrm{~N}_{2} \mathrm{O}_{2}$

$M_{r}=426.11$

Monoclinic, $P 2{ }_{1} / n$

Hall symbol: -P $2 \mathrm{yn}$

$a=4.5072(7) \AA$

$b=7.615(2) \AA$

$c=23.180(3) \AA$

$\beta=93.523(2)^{\circ}$

$V=794.1(3) \AA^{3}$

$Z=2$

\section{Data collection}

Siemens SMART 1000 CCD area-detector diffractometer

Radiation source: fine-focus sealed tube

Graphite monochromator

$\omega$ and $\varphi$ scans

Absorption correction: multi-scan

(SADABS; Sheldrick, 1996)

$T_{\min }=0.223, T_{\max }=0.514$

\section{Refinement}

Refinement on $F^{2}$

Least-squares matrix: full

$R\left[F^{2}>2 \sigma\left(F^{2}\right)\right]=0.037$

$w R\left(F^{2}\right)=0.097$

$S=1.04$

1400 reflections

100 parameters

0 restraints

Primary atom site location: structure-invariant direct methods
$F(000)=420$

$D_{\mathrm{x}}=1.782 \mathrm{Mg} \mathrm{m}^{-3}$

Mo $K \alpha$ radiation, $\lambda=0.71073 \AA$

Cell parameters from 2000 reflections

$\theta=2.6-26.7^{\circ}$

$\mu=5.11 \mathrm{~mm}^{-1}$

$T=298 \mathrm{~K}$

Needle-shaped, colorless

$0.42 \times 0.27 \times 0.15 \mathrm{~mm}$

3840 measured reflections

1400 independent reflections

1168 reflections with $I>2 \sigma(I)$

$R_{\text {int }}=0.043$

$\theta_{\text {max }}=25.0^{\circ}, \theta_{\min }=1.8^{\circ}$

$h=-5 \rightarrow 5$

$k=-8 \rightarrow 9$

$l=-19 \rightarrow 27$

Secondary atom site location: difference Fourier map

Hydrogen site location: inferred from neighbouring sites

$\mathrm{H}$-atom parameters constrained

$w=1 /\left[\sigma^{2}\left(F_{\mathrm{o}}^{2}\right)+(0.0535 P)^{2}+0.3108 P\right]$

where $P=\left(F_{\mathrm{o}}{ }^{2}+2 F_{\mathrm{c}}{ }^{2}\right) / 3$

$(\Delta / \sigma)_{\max }<0.001$

$\Delta \rho_{\max }=0.28 \mathrm{e} \AA^{-3}$

$\Delta \rho_{\min }=-0.49 \mathrm{e} \AA^{-3}$ 


\section{Special details}

Geometry. All e.s.d.'s (except the e.s.d. in the dihedral angle between two 1.s. planes) are estimated using the full covariance matrix. The cell e.s.d.'s are taken into account individually in the estimation of e.s.d.'s in distances, angles and torsion angles; correlations between e.s.d.'s in cell parameters are only used when they are defined by crystal symmetry. An approximate (isotropic) treatment of cell e.s.d.'s is used for estimating e.s.d.'s involving 1.s. planes.

Refinement. Refinement of $F^{2}$ against ALL reflections. The weighted $R$-factor $w R$ and goodness of fit $S$ are based on $F^{2}$, conventional $R$-factors $R$ are based on $F$, with $F$ set to zero for negative $F^{2}$. The threshold expression of $F^{2}>\sigma\left(F^{2}\right)$ is used only for calculating $R$-factors (gt) etc. and is not relevant to the choice of reflections for refinement. $R$-factors based on $F^{2}$ are statistically about twice as large as those based on $F$, and $R$ - factors based on ALL data will be even larger.

Fractional atomic coordinates and isotropic or equivalent isotropic displacement parameters $\left(\AA^{2}\right)$

\begin{tabular}{lllll}
\hline & $x$ & $y$ & $z$ & $U_{\text {iso }} / U_{\text {eq }}$ \\
\hline Br1 & $0.56056(10)$ & $0.86904(5)$ & $0.160074(19)$ & $0.0576(2)$ \\
N1 & $-0.2668(6)$ & $0.2442(4)$ & $0.05861(13)$ & $0.0381(7)$ \\
O1 & $-0.4716(6)$ & $0.2385(3)$ & $0.00969(11)$ & $0.0422(6)$ \\
C1 & $-0.6114(8)$ & $0.0710(5)$ & $0.00676(17)$ & $0.0396(8)$ \\
H1A & -0.7723 & 0.0722 & -0.0230 & $0.048^{*}$ \\
H1B & -0.6952 & 0.0452 & 0.0434 & $0.048^{*}$ \\
C2 & $-0.1591(8)$ & $0.3960(4)$ & $0.06447(16)$ & $0.0373(8)$ \\
H2 & -0.2198 & 0.4837 & 0.0384 & $0.045^{*}$ \\
C3 & $0.0595(7)$ & $0.4362(4)$ & $0.11157(15)$ & $0.0324(8)$ \\
C4 & $0.1868(8)$ & $0.6001(4)$ & $0.11401(15)$ & $0.0350(8)$ \\
H4 & 0.1337 & 0.6827 & 0.0857 & $0.042^{*}$ \\
C5 & $0.3929(8)$ & $0.6427(4)$ & $0.15821(16)$ & $0.0369(8)$ \\
C6 & $0.4796(9)$ & $0.5227(5)$ & $0.19990(16)$ & $0.0449(9)$ \\
H6 & 0.6198 & 0.5523 & 0.2294 & $0.054^{*}$ \\
C7 & $0.3545(9)$ & $0.3565(5)$ & $0.19728(18)$ & $0.0485(10)$ \\
H7 & 0.4098 & 0.2741 & 0.2256 & $0.058^{*}$ \\
C8 & $0.1504(9)$ & $0.3121(5)$ & $0.15356(16)$ & $0.0412(9)$ \\
H8 & 0.0719 & 0.1992 & 0.1517 & $0.049^{*}$ \\
& & & & \\
\hline
\end{tabular}

Atomic displacement parameters $\left(\AA^{2}\right)$

\begin{tabular}{lllllll}
\hline & $U^{11}$ & $U^{22}$ & $U^{33}$ & $U^{12}$ & $U^{13}$ & $U^{23}$ \\
\hline Br1 & $0.0701(4)$ & $0.0416(3)$ & $0.0597(3)$ & $-0.0158(2)$ & $-0.0076(2)$ & $-0.00700(18)$ \\
N1 & $0.0374(16)$ & $0.0381(17)$ & $0.0381(18)$ & $-0.0031(13)$ & $-0.0038(13)$ & $-0.0018(13)$ \\
O1 & $0.0438(14)$ & $0.0346(13)$ & $0.0468(15)$ & $-0.0078(11)$ & $-0.0083(12)$ & $0.0016(11)$ \\
C1 & $0.037(2)$ & $0.0389(19)$ & $0.043(2)$ & $-0.0032(16)$ & $-0.0004(16)$ & $-0.0033(16)$ \\
C2 & $0.0387(19)$ & $0.0329(19)$ & $0.040(2)$ & $0.0004(15)$ & $0.0022(16)$ & $0.0032(15)$ \\
C3 & $0.0310(18)$ & $0.0343(18)$ & $0.0323(19)$ & $0.0008(14)$ & $0.0061(14)$ & $-0.0021(14)$ \\
C4 & $0.039(2)$ & $0.0322(18)$ & $0.0337(19)$ & $0.0024(15)$ & $0.0026(15)$ & $-0.0001(14)$ \\
C5 & $0.038(2)$ & $0.0344(19)$ & $0.038(2)$ & $-0.0041(15)$ & $0.0036(16)$ & $-0.0037(15)$ \\
C6 & $0.047(2)$ & $0.049(2)$ & $0.037(2)$ & $0.0008(18)$ & $-0.0054(18)$ & $-0.0027(17)$ \\
C7 & $0.052(2)$ & $0.046(2)$ & $0.047(2)$ & $0.0059(19)$ & $-0.0024(19)$ & $0.0120(18)$ \\
C8 & $0.048(2)$ & $0.0334(19)$ & $0.043(2)$ & $-0.0055(17)$ & $0.0102(18)$ & $0.0027(16)$ \\
\hline
\end{tabular}


Geometric parameters $\left(\AA,{ }^{\circ}\right)$

\begin{tabular}{|c|c|c|c|}
\hline $\mathrm{Br} 1-\mathrm{C} 5$ & $1.881(3)$ & $\mathrm{C} 3-\mathrm{C} 8$ & $1.399(5)$ \\
\hline $\mathrm{N} 1-\mathrm{C} 2$ & $1.258(4)$ & $\mathrm{C} 4-\mathrm{C} 5$ & $1.379(5)$ \\
\hline $\mathrm{N} 1-\mathrm{O} 1$ & $1.418(4)$ & $\mathrm{C} 4-\mathrm{H} 4$ & 0.9300 \\
\hline $\mathrm{O} 1-\mathrm{C} 1$ & $1.423(4)$ & $\mathrm{C} 5-\mathrm{C} 6$ & $1.369(5)$ \\
\hline $\mathrm{C} 1-\mathrm{C} 1^{\mathrm{i}}$ & $1.521(7)$ & $\mathrm{C} 6-\mathrm{C} 7$ & $1.386(5)$ \\
\hline $\mathrm{C} 1-\mathrm{H} 1 \mathrm{~A}$ & 0.9700 & $\mathrm{C} 6-\mathrm{H} 6$ & 0.9300 \\
\hline $\mathrm{C} 1-\mathrm{H} 1 \mathrm{~B}$ & 0.9700 & $\mathrm{C} 7-\mathrm{C} 8$ & $1.368(5)$ \\
\hline $\mathrm{C} 2-\mathrm{C} 3$ & $1.457(5)$ & $\mathrm{C} 7-\mathrm{H} 7$ & 0.9300 \\
\hline $\mathrm{C} 2-\mathrm{H} 2$ & 0.9300 & $\mathrm{C} 8-\mathrm{H} 8$ & 0.9300 \\
\hline $\mathrm{C} 3-\mathrm{C} 4$ & $1.373(5)$ & & \\
\hline $\mathrm{C} 2-\mathrm{N} 1-\mathrm{O} 1$ & $110.0(3)$ & $\mathrm{C} 3-\mathrm{C} 4-\mathrm{H} 4$ & 119.8 \\
\hline $\mathrm{N} 1-\mathrm{O} 1-\mathrm{C} 1$ & $109.3(3)$ & $\mathrm{C} 5-\mathrm{C} 4-\mathrm{H} 4$ & 119.8 \\
\hline $\mathrm{O} 1-\mathrm{C} 1-\mathrm{C} 1^{\mathrm{i}}$ & $110.5(4)$ & $\mathrm{C} 6-\mathrm{C} 5-\mathrm{C} 4$ & $121.1(3)$ \\
\hline $\mathrm{O} 1-\mathrm{C} 1-\mathrm{H} 1 \mathrm{~A}$ & 109.5 & $\mathrm{C} 6-\mathrm{C} 5-\mathrm{Br} 1$ & $119.9(3)$ \\
\hline $\mathrm{C} 1-\mathrm{C} 1-\mathrm{H} 1 \mathrm{~A}$ & 109.5 & $\mathrm{C} 4-\mathrm{C} 5-\mathrm{Br} 1$ & $119.0(3)$ \\
\hline $\mathrm{O} 1-\mathrm{C} 1-\mathrm{H} 1 \mathrm{~B}$ & 109.5 & $\mathrm{C} 5-\mathrm{C} 6-\mathrm{C} 7$ & $118.8(3)$ \\
\hline $\mathrm{C} 11^{\mathrm{i}}-\mathrm{C} 1-\mathrm{H} 1 \mathrm{~B}$ & 109.5 & $\mathrm{C} 5-\mathrm{C} 6-\mathrm{H} 6$ & 120.6 \\
\hline $\mathrm{H} 1 \mathrm{~A}-\mathrm{C} 1-\mathrm{H} 1 \mathrm{~B}$ & 108.1 & $\mathrm{C} 7-\mathrm{C} 6-\mathrm{H} 6$ & 120.6 \\
\hline $\mathrm{N} 1-\mathrm{C} 2-\mathrm{C} 3$ & $120.8(3)$ & $\mathrm{C} 8-\mathrm{C} 7-\mathrm{C} 6$ & $120.7(4)$ \\
\hline $\mathrm{N} 1-\mathrm{C} 2-\mathrm{H} 2$ & 119.6 & $\mathrm{C} 8-\mathrm{C} 7-\mathrm{H} 7$ & 119.6 \\
\hline $\mathrm{C} 3-\mathrm{C} 2-\mathrm{H} 2$ & 119.6 & $\mathrm{C} 6-\mathrm{C} 7-\mathrm{H} 7$ & 119.6 \\
\hline $\mathrm{C} 4-\mathrm{C} 3-\mathrm{C} 8$ & $118.7(3)$ & $\mathrm{C} 7-\mathrm{C} 8-\mathrm{C} 3$ & $120.2(4)$ \\
\hline $\mathrm{C} 4-\mathrm{C} 3-\mathrm{C} 2$ & $118.9(3)$ & $\mathrm{C} 7-\mathrm{C} 8-\mathrm{H} 8$ & 119.9 \\
\hline $\mathrm{C} 8-\mathrm{C} 3-\mathrm{C} 2$ & $122.3(3)$ & $\mathrm{C} 3-\mathrm{C} 8-\mathrm{H} 8$ & 119.9 \\
\hline $\mathrm{C} 3-\mathrm{C} 4-\mathrm{C} 5$ & $120.4(3)$ & & \\
\hline $\mathrm{C} 2-\mathrm{N} 1-\mathrm{O} 1-\mathrm{C} 1$ & $-174.7(3)$ & $\mathrm{C} 3-\mathrm{C} 4-\mathrm{C} 5-\mathrm{Br} 1$ & $-179.8(3)$ \\
\hline $\mathrm{N} 1-\mathrm{O} 1-\mathrm{C} 1-\mathrm{C}^{\mathrm{i}}$ & $-67.7(5)$ & $\mathrm{C} 4-\mathrm{C} 5-\mathrm{C} 6-\mathrm{C} 7$ & $-0.5(6)$ \\
\hline $\mathrm{O} 1-\mathrm{N} 1-\mathrm{C} 2-\mathrm{C} 3$ & $-179.6(3)$ & $\mathrm{Br} 1-\mathrm{C} 5-\mathrm{C} 6-\mathrm{C} 7$ & $-179.5(3)$ \\
\hline $\mathrm{N} 1-\mathrm{C} 2-\mathrm{C} 3-\mathrm{C} 4$ & $175.5(3)$ & $\mathrm{C} 5-\mathrm{C} 6-\mathrm{C} 7-\mathrm{C} 8$ & $0.7(6)$ \\
\hline $\mathrm{N} 1-\mathrm{C} 2-\mathrm{C} 3-\mathrm{C} 8$ & $-2.8(5)$ & $\mathrm{C} 6-\mathrm{C} 7-\mathrm{C} 8-\mathrm{C} 3$ & $-1.6(6)$ \\
\hline $\mathrm{C} 8-\mathrm{C} 3-\mathrm{C} 4-\mathrm{C} 5$ & $-2.1(5)$ & $\mathrm{C} 4-\mathrm{C} 3-\mathrm{C} 8-\mathrm{C} 7$ & $2.3(6)$ \\
\hline $\mathrm{C} 2-\mathrm{C} 3-\mathrm{C} 4-\mathrm{C} 5$ & $179.6(3)$ & $\mathrm{C} 2-\mathrm{C} 3-\mathrm{C} 8-\mathrm{C} 7$ & $-179.4(4)$ \\
\hline $\mathrm{C} 3-\mathrm{C} 4-\mathrm{C} 5-\mathrm{C} 6$ & $1.2(5)$ & & \\
\hline
\end{tabular}

Symmetry code: (i) $-x-1,-y,-z$. 